\title{
Reviewer acknowledgement 2015
}

Gen Komaki ${ }^{*}$, Mutsuhiro Nakao ${ }^{2}$ and Shin Fukudo ${ }^{3}$

\section{Contributing reviewers}

The BioPsychoSocial Medicine editorial team would like to thank all reviewers who have contributed to the journal in Volume 9 (2015).

Tetsuya Ando
Japan

Shuji Aou

Japan

Kashif Aziz

Pakistan

Hans-Christian Deter

Germany

Shin Fukudo

Japan

Mikihiko Fukunaga

Japan

Hirokazu Furukawa

Japan

Yusuke Hada

Japan

Masahiro Hashizume

Japan

Hirono Ishikawa

Japan

Yuko Ishizaki

Japan

Motoyori Kanazawa

Japan

Kenji Kanbara

Japan
Michiko Kano
Japan

Hiroe Kikuchi

Japan

Masayo Kojima

Japan

Hiroaki Kumano

Japan

María Torres Lacomba

Spain

Mariko Makino

Japan

Astrid Müller

Germany

Masanori Munakata

Japan

Shinichiro Nagamitsu

Japan

Jun Nagano

Japan

Toshihiko Nagata

Japan

Mutsuhiro Nakao

Japan

Shinobu Nomura

Japan
Yuko Odagiri

Japan

Mariko Ogawa

Japan

Kyoko Ohashi

United States

Takakazu Oka

Japan

Hirokuni Okumi

Japan

Yoshifumi Okura

Japan

Annelieke Roest

Netherlands

Amy Ronaldson

United Kingdom

Ken Shimizu

Japan

Hideaki Soya

Japan

Nobuyuki Sudo

Japan

Nagisa Sugaya

Japan

Shu Takakura

Japan

\footnotetext{
* Correspondence: komaki@iuhw.ac.jp

${ }^{1}$ International University of Health and Welfare, School of Health Sciences,

Fukuoka 831-8501, Japan

Full list of author information is available at the end of the article
} 
Takeaki Takeuchi

Japan

Masato Takii

Japan

Tetsushi Tamekazu

Japan

Yoshihisa Wada

Japan
Jong Min Woo

South Korea

Kazufumi Yoshihara

Japan

Kazuhiro Yoshiuchi

Japan

Stephan Zipfel

Germany
Author details

International University of Health and Welfare,

School of Health Sciences, Fukuoka 831-8501,

Japan. ${ }^{2}$ Teikyo University Graduate School of

Public Heath, Tokyo 173-8605, Japan. ${ }^{3}$ Tohoku

University Graduate School of Medicine, Sendai

980-8575, Japan. 